\title{
A Maglev System: Modeling and Controller Design
}

\author{
E. Alvarez-Sanchez, Ja. Alvarez-Gallegos and R. Castro-Linares
}

\begin{abstract}
In this paper, the nonlinear mathematical model with five DOFs (degrees-of-freedom) of a magnetic levitation system is developed and analyzed. Then a second order sliding mode controller is proposed to regulate the levitation to a desired position, stabilize the other 4 DOFs in the nonlinear system and compensate the unknown increments on the load. Simulation results are presented to show the effectiveness of the proposed controller.
\end{abstract}

\section{INTRODUCTION}

The transport of material or products is a major problem in the manufacturing automation industry. As it currently stands transport specifications can be so variable from process within a single plant that each operation might require its own transport. Using magnetic levitation (maglev), a carrier can be partially or totally levitated or suspended by magnetic fields generated along the guiding tracks. This allows the carrier to move with little or no contact to the guiding tracks, thus greatly minimizing the problems of environmental contamination. Of course, such contact-free levitation has to be enforced for all DOFs of the rigid body.

Maglev systems offer many advantages such as frictionless, low noise, the ability to operate in high vacuum environments and so on. Previous works in this area span many fields. Some well known fields include maglev transportation [1], microrobotics [2], photolitography [3], positioning [4], launch systems [5] and so on.

In general a maglev system can be classified, based on the levitation forces, as an attractive system or a repulsive one, each type having various kinds of possible arrangements. Most of the maglev systems discussed in the literature are attractive, where attractive forces are applied between the moving carriage and fixed guide tracks. On the other hand, the repulsive maglev systems use repulsive forces to push the moving carriage above the fixed guide tracks. However, a magnetic levitation system is highly nonlinear and unstable, and a feedback control is necessary to achieve a stable operation. Many works have developed linear controllers, and the control laws have been based on traditional control methods and only local stability is guaranteed. These developed controllers may not meet the precision control purpose for maglev systems, because these systems are naturally under the influence of many uncertainties. On the other hand, the works that use nonlinear mathematical models [6] only control 2 DOFs and consider the other DOFs stables.

To overcome this problem, a new approach called "second order sliding mode (SOSM)" has been proposed [7], [8], [9].

CINVESTAV-IPN, Department of Electrical Engineering, Av. IPN No. 2508, Col. San Pedro Zacatenco, 07360 Mexico, D.F., Mexico ejalvare, jalvarez, rcastro@cinvestav.mx
This approach has the main advantages of the standard sliding mode control technique, the chattering effect is eliminated and a high order precision is provided.

In this paper the kind of maglev system is a repulsive one, using an arrangement of a permanent magnet levitated above an electromagnet. The control design proposed here is based on SOSM control technique for the nonlinear maglev mathematical model; this controller is robust when different loads are put on the carrier and guarantees stabilization and precision positioning.

The organization of this paper is as follows. In section II, the maglev system is described, some magnetics formulas will be reviewed and the mathematical model is obtained. In section III a SOSM controller is designed using the nonlinear system obtained. Section IV presents numerical simulations results that show the robustness of the controller designed. Finally conclusions are given in section V.

\section{SYSTEM MODELING}

In this section, the mechanical structure of a Maglev system will be described. Its analytical model of 5 DOFs will be derived and analyzed. The 6th DOF, propulsion in the $y$ direction, will be analyzed and controlled in a future work. The overall system is shown in Fig.1.

\section{A. The maglev system}

Basically, the maglev system proposed here is a multi-input multi-output (MIMO) system. Here, the states are the lateral and vertical displacement, $x$ and $z$ respectively, and the three rotations $\theta, \psi$ and $\phi$. The outputs are $x, \theta, z, \psi$ and $\phi$ while the inputs are the currents applied to coils into the levitation guiding tracks. The dynamics of the maglev system can be divided into a stable part and an unstable one. The stable part consists of the dynamic of $z$, while the unstable part consists of the dynamics of $x, \theta, \psi$ and $\phi$.

In order to control 3 DOFs in rotational displacement and 2 DOFs in lateral displacement, i.e., a total of 5 DOFs of the carrier separately, a four-track design, shown in Fig.2,

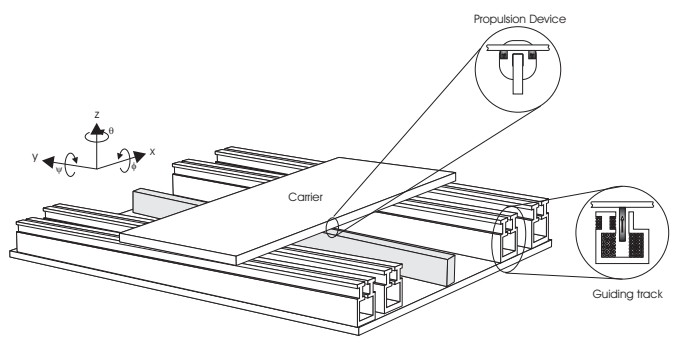

Fig. 1. 3D view of maglev system 
is sufficient to supply such 5-DOFs control. The guiding tracks together must provide a levitation force to counteract the carrier weight. On the other hand, to provide a uniform magnetic field along the guiding tracks, an oblong coil is necessary.

Also, due to the nature of lateral instability of a repulsive system, stabilizers are needed inside.The stabilizers can control the lateral position of a levitating $\mathrm{NdFeB}$ magnet whereas the levitator can control the vertical (up-down) position of a levitating $\mathrm{NdFeB}$ magnet.

The four stabilizing coils are grouped into two sets: inner guiding tracks and outer guiding tracks. Then, the principle shown in Figs. 3 and 4 to control the rotation of the carrier about a vertical axis and its lateral translation can be used.

In order to obtain the magnetic force in $z$ and $x$ is necessary to analyze some magnetic formulas. According to Biot-Savart's law and Ampere's circuit law [10] the magnetic flux density in any point around an infinitely long currentcarrying straight wire at a point $(x, z)$ can be obtained as

$$
\mathbf{B}=\frac{\mu_{0} I}{2 \pi}\left[\frac{-z}{x^{2}+z^{2}} \hat{i}+\frac{x}{x^{2}+z^{2}} \hat{k}\right]
$$

where $\mu_{0}$ is the permeability of free space, $I$ is the input current, $\hat{i}$ and $\hat{k}$ are the unit vector in the Cartesian coordinate.

If we deal with a permanent magnet as a single dipole moment, the expression of the Lorentz force $\mathbf{F}$ exerted on the permanent magnet by an external magnetic field $\mathbf{B}$ can be characterized by the following vector equation

$$
\mathbf{F}=(\mathbf{u} \cdot \nabla) \mathbf{B}
$$

where $\mathbf{u}$ is the dipole moment of the permanent magnet. Assuming that the dipole lies in the $z$ direction, useful scalar equations of the force components can be derived from (2) as

$$
\begin{aligned}
& F_{x}=\frac{\mu_{0} I u_{z}}{2 \pi} \frac{\left(z^{2}-x^{2}\right)}{\left(x^{2}+z^{2}\right)^{2}} \\
& F_{z}=\frac{\mu_{0} I u_{z}}{\pi} \frac{-x z}{\left(x^{2}+z^{2}\right)^{2}}
\end{aligned}
$$

\section{B. Nonlinear model}

Consider a carrier represented by an uniform box-shaped object with the center of mass coincident with the center of geometry. The principle of linear momentum leads to the following equations:

$$
F_{x}=m \ddot{x}, F_{z}=m \ddot{z}
$$

where $F_{x}$ and $F_{z}$ are the resultant forces acting on the carrier along the $\mathrm{x}$-axis and $\mathrm{z}$-axis, respectively, and $\mathrm{m}$ is the mass of the carrier.

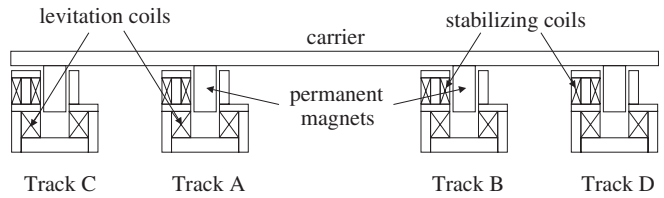

Fig. 2. Front view of the maglev system

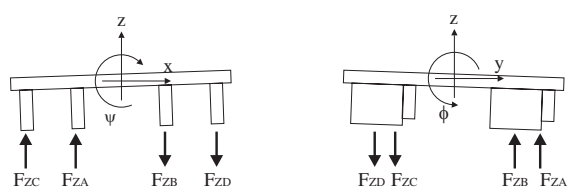

Fig. 3. Magnetic levitation forces

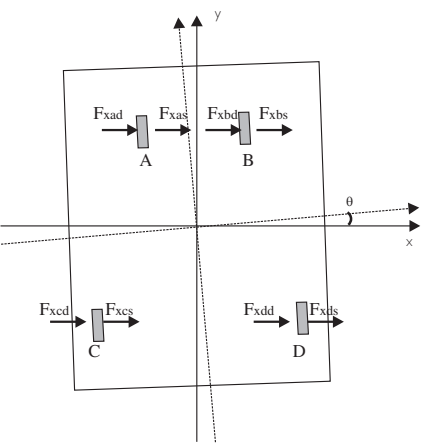

Fig. 4. Destabilizing and stabilizing forces

By the same token, the principle of angular momentum leads to torque equations for the rotational coordinates

$$
T_{z}=J_{z} \theta, T_{y}=J_{y} \psi, T_{x}=J_{x} \phi
$$

where $T_{z}, T_{y}$ and $T_{x}$ are the external torques, $J_{z}, J_{y}$ and $J_{x}$ are the principal moments of inertia and $\theta, \psi$ and $\phi$ are the three angular rotation of the rigid body.

To understand the dynamics of the maglev system it is necessary to describe an arbitrary orientation of the carrier in space. This orientation can be obtained using the Euler angular description $\operatorname{yaw}(\theta)$-roll $(\psi)$ - $\operatorname{pitch}(\phi)$ given by the following rotation matrix

$$
R=\left[\begin{array}{ccc}
c \theta c \psi & c \theta s \psi s \phi-s \theta c \phi & c \theta s \psi c \phi+s \theta s \phi \\
s \theta c \psi & s \theta s \psi s \phi+c \theta c \phi & s \theta s \psi c \phi-c \theta s \phi \\
-s \psi & c \psi s \phi & c \psi c \phi
\end{array}\right]
$$

where $c$ and $s$ represents $\cos$ and $\sin$ respectively. The position of the levitation magnets on the carrier are

$$
\begin{array}{rr}
A\left(-b_{1}, a, 0\right) & B\left(b_{1}, a, 0\right) \\
C\left(-b_{2},-a, 0\right) & D\left(b_{2},-a, 0\right)
\end{array}
$$

where $A, B, C$ and $D$ denote the center position of these magnets and $a, b_{1}$ and $b_{2}$ are known dimensions. If one assumes small pitch, roll and yaw angle for the carrier, in additional to $x$ and $z$ translation, the position of the magnets on the carrier can be calculated as

$$
\begin{aligned}
b_{(x, y, z) a}= & {\left[\begin{array}{c}
-b_{1}-a \theta+x \\
a-b_{1} \theta \\
b_{1} \psi+a \phi+z
\end{array}\right] } \\
b_{(x, y, z) b}= & {\left[\begin{array}{c}
b_{1}-a \theta+x \\
a+b_{1} \theta \\
-b_{1} \psi+a \phi+z
\end{array}\right] } \\
b_{(x, y, z) c}= & {\left[\begin{array}{c}
-b_{2}+a \theta+x \\
-a-b_{2} \theta \\
b_{2} \psi-a \phi+z
\end{array}\right] }
\end{aligned}
$$




$$
b_{(x, y, z) d}=\left[\begin{array}{c}
b_{2}+a \theta+x \\
-a+b_{2} \theta \\
-b_{2} \psi-a \phi+z
\end{array}\right]
$$

In similar form, the positions of the magnets into the guiding tracks, are obtained as

$$
\begin{aligned}
& {\left[\begin{array}{l}
x_{a, b} \\
y_{a, b} \\
z_{a, b}
\end{array}\right]=\left[\begin{array}{c}
-a \theta+d_{l} \psi+x \\
a-d_{l} \phi \\
a \phi+d_{l}+z
\end{array}\right]} \\
& {\left[\begin{array}{l}
x_{c, d} \\
y_{c, d} \\
z_{c, d}
\end{array}\right]=\left[\begin{array}{c}
a \theta+d_{l} \psi+x \\
-a-d_{l} \phi \\
-a \phi+d_{l}+z
\end{array}\right]}
\end{aligned}
$$

Before formulating equations of motion, some notations on force and distances are explained in the following: the force subscript has two letters, the first means the label of the magnet, and the second the magnetic force type, $d$ for destabilizing, $s$ for stabilizing and $l$ for levitation, e.g. $F_{a d}$ is the destabilizing force applied to the magnet A caused by the levitation coil A . The position of the magnets, denoted by $b$, has a subscript with two letters, the first means the direction of the distance and the second the label of the magnet, e.g. $b_{x a}$ is the distance in the $x$ direction from the carrier center to the center of the magnet $\mathrm{A}$.

By substituting the positions of the levitation magnet (9) into the magnetic force equations (3) and (4) one can get the forces exerted on each levitation magnet. Next, one can substitute these force equations into the dynamics of the carrier, and then the equations of motion can be obtained as

$$
\begin{aligned}
m_{t} \ddot{x}= & F_{a d} I_{a}+F_{a s} I_{s 1}+F_{b d} I_{b}+F_{b s} I_{s 1} \\
& +F_{c d} I_{c}+F_{c s} I_{s 2}+F_{d d} I_{d}+F_{d s} I_{s 2} \\
J_{z} \ddot{\theta}= & -b_{y a} F_{a d} I_{a}-b_{y a} F_{a s} I_{s 1}-b_{y b} F_{b d} I_{b} \\
& -b_{y b} F_{b s} I_{s 1}+b_{y c} F_{c d} I_{c}+b_{y c} F_{c s} I_{s 2} \\
& +b_{y d} F_{d d} I_{d}+b_{y d} F_{d s} I_{s 2} \\
m_{t} \ddot{z}= & F_{a l} I_{a}+F_{b l} I_{b}+F_{c l} I_{c}+F_{d l} I_{d} \\
& +F_{p}-m_{t} g \\
J_{y} \ddot{\psi}= & b_{x a}\left(F_{a l} I_{a}-w_{a}\right)-b_{x b}\left(F_{b l} I_{b}-w_{b}\right) \\
& +b_{x c}\left(F_{c l} I_{c}-w_{c}\right)-b_{x d}\left(F_{d l} I_{d}-w_{d}\right) \\
& -b_{z a} F_{a d} I_{a}-b_{z a} F_{a s} I_{s 1}-b_{z b} F_{b d} I_{b} \\
& -b_{z b} F_{b s} I_{s 1}-b_{z c} F_{c d} I_{c}-b_{z c} F_{c s} I_{s 2} \\
& -b_{z d} F_{d d} I_{d}-b_{z d} F_{d s} I_{s 2} \\
& b_{y a}\left(F_{a l} I_{a}-w_{a}\right)+b_{y b}\left(F_{b l} I_{b}-w_{b}\right) \\
& -b_{y c}\left(F_{c l} I_{c}-w_{c}\right)-b_{y d}\left(F_{d l} I_{d}-w_{d}\right) \\
J_{x} \ddot{\phi}= &
\end{aligned}
$$

where $g$ is the acceleration due to gravity, $m_{t}$ is the mass of the carrier and load, $J$ represents the moment of inertia of the carrier, $w$ represents the weight above each magnet, $I_{s 1}$ is the current in the inner stabilizer whereas $I_{s 2}$ is the current in the outer stabilizer. $I_{a}, I_{b}, I_{c}, I_{d}$ represent the currents in the levitators corresponding to the levitation magnets A, B, C and D respectively. $F_{p}$ represents the damping force produced by the levitation coils and can be modeled as $F_{p}=-K_{d a m} \dot{z}$, where $K_{d a m}$ is a positive constant.

\section{CONTROLLER DESIGN}

In this section, a control scheme is presented for the levitation an stabilization dynamics of the magnetic system described in section 2. The aim is to control the height $z$ while the lateral and rotational displacements are tried to be kept near to zero. For doing this a SOSM proposed in [7] is applied to the nonlinear model (10). For symplicity, the currents in the levitation coils A and B are set to be same, thus $I_{a b}=I_{a}=I_{b}$. One also defines the state vector $\rho=$ $\left[\begin{array}{llllllll}x & \dot{x} & \theta & \dot{\theta} & z & \dot{z} & \psi & \dot{\psi}\end{array} \phi \dot{\phi}\right]^{T}$ together with the input vector $u=$ $\left[\begin{array}{lllll}I_{a b} & I_{c} & I_{d} & I_{s 1} & I_{s 2}\end{array}\right]^{T}$ and the output vector $y=\left[\begin{array}{llll}x & \theta & z & \psi\end{array}\right]^{T}$. The nonlinear model (10) can then be rewritten in the state space form

$$
\begin{aligned}
\dot{\rho} & =f(\rho)+\Delta f(\rho)+\sum_{i=1}^{5}\left[g_{i}(\rho)+\Delta g_{i}(\rho)\right] u_{i} \\
y & =h(\rho)
\end{aligned}
$$

where

$$
\begin{aligned}
& f(\rho)=\operatorname{col}\left[\dot{x}, 0, \dot{\theta}, 0, \dot{z},\left(-g-\frac{K_{d a m} \dot{z}}{m_{t}}\right),\right. \\
& \dot{\psi}, 0, \dot{\phi}, 0] \\
& \Delta f(\rho)=\operatorname{col}\left[0,0,0,0,0, \Delta f_{6}(\rho), 0,0,0,0\right] \\
& g_{1}(\rho)=\left[\begin{array}{c}
0 \\
\frac{F_{a d}+F_{b d}}{m_{t}} \\
0 \\
\frac{F_{a l}+F_{b l}}{m_{t}} \\
0 \\
\frac{-b_{y a} F_{a d}-b_{y b} F_{b d}}{J_{z}} \\
0 \\
\frac{b_{x a} F_{a l}-b_{x b} F_{b l}-b_{z a} F_{a d}-b_{z b} F_{b d}}{J_{y}} \\
0 \\
\frac{b_{y a} F_{a l}+b_{y b} F_{b l}}{J_{x}}
\end{array}\right] \\
& g_{2,3}(\rho)=\left[\begin{array}{cc}
0 & 0 \\
\frac{F_{c d}}{m_{t}} & \frac{F_{d d}}{m_{t}} \\
0 & 0 \\
\frac{F_{c l}}{m_{t}} & \frac{F_{d l}}{m_{t}} \\
0 & 0 \\
\frac{b_{y c} F_{c d}}{J_{z}} & \frac{b_{y d} F_{d d}}{J_{z}} \\
0 & 0 \\
\frac{b_{x c} F_{c l}-b_{z c} F_{c d}}{J_{y}} & \frac{b_{x d} F_{d l}-b_{z d} F_{d d}}{J_{y}} \\
0 & 0 \\
\frac{-b_{y c} F_{c l}}{J_{x}} & \frac{-b_{y d} F_{d l}}{J_{x}}
\end{array}\right]
\end{aligned}
$$




$$
\begin{aligned}
& g_{4,5}(\rho)=\left[\begin{array}{cc}
0 & 0 \\
\frac{F_{a e}+F_{b e}}{m_{t}} & \frac{F_{c e}+F_{d e}}{m_{t}} \\
0 & 0 \\
0 & \frac{F_{c e}+F_{d e}}{m_{t}} \\
0 & 0 \\
\frac{-b_{y a} F_{a e}-b_{y b} F_{b e}}{J_{z}} & \frac{b_{y c} F_{c e}+b_{y d} F_{d e}}{J_{z}} \\
0 & 0 \\
\frac{-b_{z a} F_{a e}-b_{z b} F_{b e}}{J_{y}} & \frac{-b_{z c} F_{c e}-b_{z d} F_{d e}}{J_{y}} \\
0 & 0 \\
0 & 0
\end{array}\right] \\
& \Delta g_{j}(\rho)=\left[\begin{array}{c}
\Delta g_{1 j}(\rho) \\
\vdots \\
\Delta g_{10 j}(\rho)
\end{array}\right] \quad j=1, \ldots, 5 \\
& h(\rho)=\left[\begin{array}{lllll}
\rho_{1} & \rho_{3} & \rho_{5} & \rho_{7} & \rho_{9}
\end{array}\right]^{T}
\end{aligned}
$$

$\Delta f(\rho)$ and $\Delta g_{j}(\rho)$ represent modeling uncertainties associated to the magnetic system. Equation (11) can also be written in a more condensed form as

$$
\begin{aligned}
\dot{\rho} & =f(\rho)+\Delta f(\rho)+[G(\rho)+\Delta G(\rho)] u \\
y & =h(\rho)
\end{aligned}
$$

where

$$
\begin{aligned}
G(\rho) & =\left[\begin{array}{lll}
g_{1}(\rho) & \ldots & g_{5}(\rho)
\end{array}\right] \\
\Delta G(\rho) & =\left[\begin{array}{llll}
\Delta g_{1}(\rho) & \ldots & \Delta g_{5}(\rho)
\end{array}\right]
\end{aligned}
$$

The goal is to make the output $y(\rho)$ in system (12) follow a desired trajectory $y_{d}(t)$. The control strategy should be robust enough to handle the modeling uncertainties $\Delta f$ and $\Delta G$. The upper bounds of these equations are assumed to be

$$
\begin{aligned}
\left|\Delta f_{6}(\rho)\right| & \leq \sigma(\rho) \\
\left|\Delta g_{i j}(\rho)\right| & \leq \alpha_{i j}(\rho) \quad i=1, \ldots, 10 \\
&
\end{aligned}
$$

When the modeling uncertainties are not considered, this is $\Delta f(\rho)=0$ and $\Delta G(\rho)=0$, one has the exact model

$$
\begin{aligned}
\dot{\rho} & =f(\rho)+G(\rho) u \\
y & =h(\rho)
\end{aligned}
$$

for which one can easily verify, in accordanace to [11] that it has a (vector) relative degree $\left[r_{1}, r_{2}, r_{3}, r_{4}, r_{5}\right]=[2,2,2,2,2]$ at a point $\rho_{0}=0$. In particular the decoupling matrix $A(\rho)$ is given by

$$
A(\rho)=\left[\begin{array}{ccc}
a_{11} & \cdots & a_{15} \\
\vdots & \ddots & \vdots \\
a_{51} & \cdots & a_{55}
\end{array}\right]
$$

where

$$
a_{i j}=L_{g_{j}} L_{f} h_{i}=g_{(2 i) j}(\rho) \quad i, j=1, \ldots, 5
$$

which is nonsingular at $\rho=0$. One can also verify that, for the uncertain system (12),

$$
\begin{array}{ll}
\Delta f(\rho) & \text { and } \\
\Delta G(\rho) & \in \quad \operatorname{Ker}\left[d h_{i}, d L_{f} h_{i}, \ldots, d L_{f}^{r_{i}-2} h_{i}\right]
\end{array}
$$

for $i=1, \ldots, 5$. This is, the so-called matching condition is achieved. Thus the uncertainties $\Delta f$ and $\Delta G$ do not appear in the time derivatives of $y_{i}$ of order less than $r_{i}=2$ and the (vector) relative degree is unchanged. Besides, since $\sum_{i=1}^{5} r_{i}=10$, system (12) has no unobservable internal dynamics. Following [7], a SOSM strategy that allows to have reference output tracking despite the presence of the uncertainties can be obtained by setting

$$
\dot{s_{j}}+z_{0} s_{j}=\dot{e}_{j}+c_{j 1} e_{j}+c_{j 0} \int e_{j} \quad j=1, \ldots, 5
$$

where $s_{j}=\dot{s_{j}}=0$ represents the $j$ th sliding surface and $e_{j}=y_{j}-y_{j d}$ is the $j$ th tracking error, with $y_{j d}$ being the $j$ th component of the desired output $y_{d}$. The constant real coefficients $c_{j 0}$ and $c_{j 1}$ are chosen in such a way that the polynomial $\pi^{2}+c_{j 1} \pi+c_{j 0}=0$ is Hurtwitz. $z_{0}$ is also a constant real coefficient.

By choosing a Lyapunov function candidate as

$$
V_{j}=\frac{1}{2}\left(\dot{s}^{T} \dot{s}+\omega_{n}^{2} s^{T} s\right) \quad \text { for } j=1, \ldots, 5
$$

where $s=\left[s_{1}, \ldots, s_{5}\right]^{T}$ and $\omega_{n}$ is a real coefficient, one has that, the Lyapunov stability criterion leads to the condition

$$
\dot{s}^{T}\left(\ddot{s}+\omega_{n}^{2} s\right) \leq 0
$$

which is known as the attractivity condition towards $s=\dot{s}=$ 0 . By setting

$$
\ddot{s}=-K \operatorname{sgn}(\dot{s})-\omega_{n}^{2}
$$

where $K$ is a real positive number different from zero and $\operatorname{sgn}(\dot{s})=\left[\operatorname{sgn}\left(\dot{s}_{1}\right), \ldots, \operatorname{sgn}\left(\dot{s}_{5}\right)\right]^{T}$, one can assures the fulfillment of condition (19). From this last equation and considering the exact model (14) one has the sliding control $u=u_{s}$ given by

$$
\begin{aligned}
u_{s}= & -A^{-1}(\rho)\left[F(\rho)+C E-y_{d}^{(r)}-z_{0} \dot{s}\right] \\
& -A^{-1}(\rho)\left[K \operatorname{sgn}(\dot{s})+\omega_{n}^{2} s\right]
\end{aligned}
$$

where

$$
\begin{aligned}
& F(\rho)= {\left[\begin{array}{c}
L_{f}^{2} h_{1} \\
L_{f}^{2} h_{2} \\
L_{f}^{2} h_{3} \\
L_{f}^{2} h_{4} \\
L_{f}^{2} h_{5}
\end{array}\right]=\left[\begin{array}{c}
0 \\
0 \\
-g-\frac{K_{d a m}}{m_{t}} \rho_{6} \\
0 \\
0
\end{array}\right] } \\
& C E= {\left[\begin{array}{c}
c_{10} e_{1}+c_{11} \dot{e}_{1} \\
\vdots \\
c_{50} e_{5}+c_{51} \dot{e}_{5}
\end{array}\right] } \\
& y_{d}^{r}=\left[\begin{array}{lll}
\ddot{y}_{d 1} & \ldots & \ddot{y}_{d 5}
\end{array}\right]^{T}
\end{aligned}
$$


For the uncertain system (12) (this is $\Delta f \neq 0$ and $\Delta G \neq$ 0 ), when the sliding control $u_{s}$ is substituted into (19), the attractivity condition takes the form

$$
\begin{aligned}
& \dot{s}^{T}\left[-K\left(I+\Delta A(\rho) A^{-1}(\rho)\right) \operatorname{sgn}(\dot{s})\right. \\
+ & \left.\Delta A(\rho) A^{-1}(\rho)(\widehat{v}-F(\rho))+\Delta F(\rho)\right]<0
\end{aligned}
$$

where

$$
\begin{aligned}
\Delta A(\rho)= & {\left[\begin{array}{c}
L_{\Delta g_{j}} L_{f} h_{1} \\
\vdots \\
L_{\Delta g_{j}} L_{f} h_{5}
\end{array}\right] \quad j=1, \ldots, 5 } \\
\Delta F(\rho) & =\left[\begin{array}{l}
L_{\Delta f} L_{f} h_{1} \\
L_{\Delta f} L_{f} h_{2} \\
L_{\Delta f} L_{f} h_{3} \\
L_{\Delta f} L_{f} h_{4} \\
L_{\Delta f} L_{f} h_{5}
\end{array}\right]=\left[\begin{array}{c}
0 \\
0 \\
\Delta f_{6} \\
0 \\
0
\end{array}\right] \\
\widehat{v} & =y_{d}^{r}-C E+z_{0} \dot{s}-\omega_{n}^{2} s
\end{aligned}
$$

One can notice that $\dot{s}^{T} \operatorname{sgn}(\dot{s}) \geq\|\dot{s}\|$, thus $-K \dot{s}^{T} \operatorname{sgn}(\dot{s}) \leq-K\|\dot{s}\|$, and (22) can be reiterated using vector norms obtaining

$$
\begin{gathered}
-K+K\left\|\Delta A A^{-1} \operatorname{sgn}(\dot{s})\right\| \\
+\left\|\Delta A A^{-1}(\widehat{v}-F)\right\|+\|\Delta F\| \leq-\mu
\end{gathered}
$$

where $\mu>0$. This last expression leads to the following

$$
K \geq \frac{\left\|\Delta A A^{-1}(\widehat{v}-F)\right\|+\|\Delta F\|+\mu}{1-\left\|\Delta A A^{-1} \operatorname{sgn}(\dot{s})\right\|}
$$

were it is assumed that $\left\|\Delta A A^{-1} \operatorname{sgn}(\dot{s})\right\|<1$.

\section{SIMULATION RESULTS}

In this section, a series of simulation are proposed for the maglev system using the SOSM controller designed in the previous section. The simulation parameters are listed in table I, these values are proposed usign the real dimensions of the permanent magnets that will be used in the maglev prototipe that is being built in our laboratory. The desired values for all states are equal to zero, this means that the permanent magnets are regulated at the center of the guiding tracks whereas the carrier is located in the center of the system. To avoid the discontinuity, a saturation function sat (dots) is replaced instead of $\operatorname{sgn}(\mathrm{dots})$ as follows

$$
\operatorname{sat}(\dot{s})=\left\{\begin{array}{cc}
1 & \dot{s}>\epsilon \\
\dot{s} & -\epsilon \leq \dot{s} \geq \epsilon \\
-1 & \dot{s}<-\epsilon
\end{array}\right\}
$$

The first simulation was made with no load perturbations and with the initial conditions $x(0)=2 \mathrm{~mm}, \theta(0)=25 \mathrm{mrad}$, $z(0)=-8 \mathrm{~mm}, \psi(0)=35 \mathrm{mrad}$ and $\phi(0)=-25 \mathrm{mrad}$. Figures 5 and 6 show that all the states converge to zero as t goes to infinity. Figures 7 and 8 show the levitation and stabilization control currents, respectively. In Fig.8 one can notice that the stabilization control currents have a zero value at steady state, this means that permanent magnets are located at the center of the guiding tracks, where the destabilizing forces are equal to zero.

The second simulation tests the capability of load disturbance rejection. The load disturbance is applied on the carrier over each levitation magnet (see figures 3 and 4) after the carrier reaches a steady-state. Disturbances of $0.25 \mathrm{Kg}$ are introduced at $0.3 \mathrm{~s}, 0.6 \mathrm{~s}, 1 \mathrm{~s}$ and $1.5 \mathrm{~s}$. In this case, the initial conditions were $x(0)=2 \mathrm{~mm}, \theta(0)=2.5 \mathrm{mrad}, z(0)=$ $-8 \mathrm{~mm}, \mathrm{psi}(0)=3.5 \mathrm{mrad}$ and $\phi(0)=-2.5 \mathrm{mrad}$. Figures 9 and 10 show the response of the maglev system when a load disturbance is applied on the carrier. One can observe that all the states go to equilibrium points when the load disturbance increases. Fig.11 shows the changes in the levitation control currents due to different load disturbances; one can observe the increments or decrements in the current magnitude after the load disturbance increases. Fig.12 shows that the currents in both stabilizers do not present any change, this is because the load does not affect the $x$ translation and the $\theta$ rotation.

\section{CONCLUSIONS}

In this paper a nonlinear mathematical model for a maglev system has been derived. A repulsive maglev system with four guiding tracks is adopted here. There, the maglev system has been treated as a MIMO system, and a SOSM controller for a nonlinear 5 DOFs maglev system has been designed here. From the simulation results, the feasibility and effectiveness of the designed controller have been clearly shown. The desired performances of levitation and lateral and rotational stabilization have been achieved.

\section{REFERENCES}

[1] Y. Luguang, "Progress of High-Speed Maglev in China,"IEEE Transactions on Applied Superconductivity, vol. 12, no. 1, pp. 944-947, March 2002.

[2] M. B. Khamesse, N. Kato, Y. Kamura and T. Nakamura, "Design and Control of a Microrobotic System using Magnetic Levitation,'IEEE/ASME Transactions on Mechatronics, vol. 7, no. 1, pp. 1-14, March 2002.

[3] W. J. Kim and D. L. Trumper, "High-Precision Magnetic Levitation Stage for Photolithography,"Precision Engineering, vol. 22, no. 2, pp. 66-77, April 1998.

[4] K. Suk and Y. S. Baek, "Contact-Free Moving-Magnet Type of Micropositioner with opimized specification,"IEEE Transactions on Magnetics, vol. 38, no. 3, pp. 1539-1548, May 2002.

[5] W. A. Jacobs, "Magnetic Launch Assist-NASA's Vision for the Future,'IEEE Transactions on Magnetics, vol. 37, no. 1, pp. 55-57, January 2001.

[6] J. Kaloust, C. Ham, J. Siehling, E. Jongekryg and Q. Han, "Nonlinear Robust Control Design for Levitation and Propulsion of a Maglev System,"IEE Proc. Control Theory Appl., vol. 151, no.4, pp. 460-464, July 2004

[7] H. Elmali and N. Olgac, "Robust Output Tracking Control of Nonlinear MIMO Systems via Sliding Mode Technique,"Automatica, vol. 28, no. 1, pp. 145-151, 1992

[8] G. Bartolini, A. Ferrara, A. Pisano and E. Usai, "On the Convergence Properties of a 2-Sliding Control Algorithm for Non-Linear Uncertain Systems,"International Journal of Control, vol. 74, no.7, pp. 718-731, 2001

[9] R. Castro-Linares and A. Glumineau and S. Laghraouche and F. Plestan, "Higher Order Siding Mode Oberver-Based Control," in2nd Symposium on System, Structure and Control, Oaxaca, Mexico, pp. 517-522, 2004

[10] M. H. Nayef and M. K. Brussel, Electricity and Magnetism. New York: John Wiley and Sons, Inc., 1985

[11] A. Isidori, Nonlinear Control Systems. New York: Springer Verlag, 1995 
TABLE I

SYSTEM PARAMETERS

\begin{tabular}{|c|c|}
\hline \hline Mass m & $1.0536 \mathrm{Kg}$ \\
\hline Carrier dimension & $405 \times 205 \times 6 \mathrm{~mm}$ \\
\hline NdFeB Size & $13.91 \times 31.62 \times 25.4 \mathrm{~mm}$ \\
\hline NdFeB Br & $1.19 \mathrm{~T}$ \\
\hline Turns of levitator & 240 turns \\
\hline Turns of stabilizer & 120 turns \\
\hline$a, b_{1}, b_{2}$ & $70,81,175 \mathrm{~mm}$ \\
\hline$d_{e}, d_{d}, d_{l}$ & $12,15,17 \mathrm{~mm}$ \\
\hline$c_{10}, c_{20}, c_{30}, c_{40}, c_{50}$ & $2,2,1,2,2$ \\
\hline$c_{11}, c_{21}, c_{31}, c_{41}, c_{51}$ & $50,40,40,50,50$ \\
\hline$K, K_{d a m}$ & 170,27 \\
\hline$\omega_{n}, z_{0}$ & $75,0.7$ \\
\hline \hline
\end{tabular}

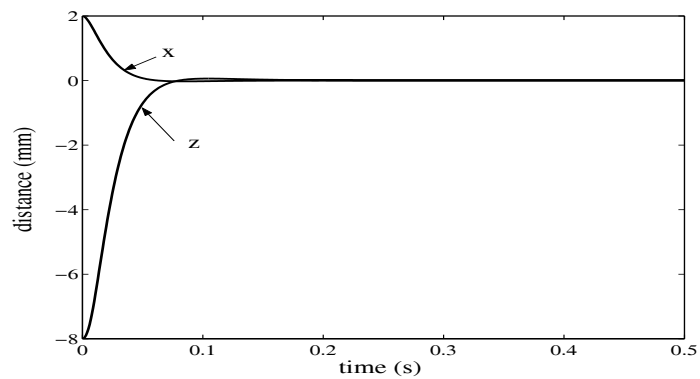

Fig. 5. Carrier motion in $x$ and $z$ without load disturbances

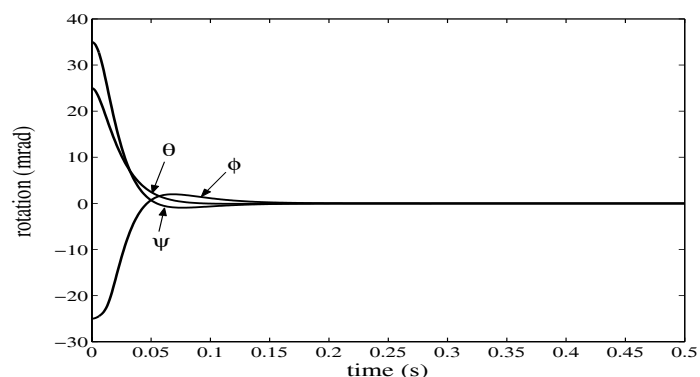

Fig. 6. Carrier rotations $\theta, \psi$ and $\phi$ without load disturbances

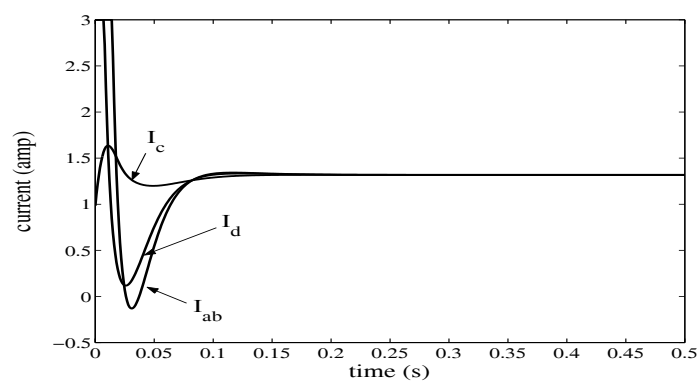

Fig. 7. Levitation currents A, C and D without load disturbances

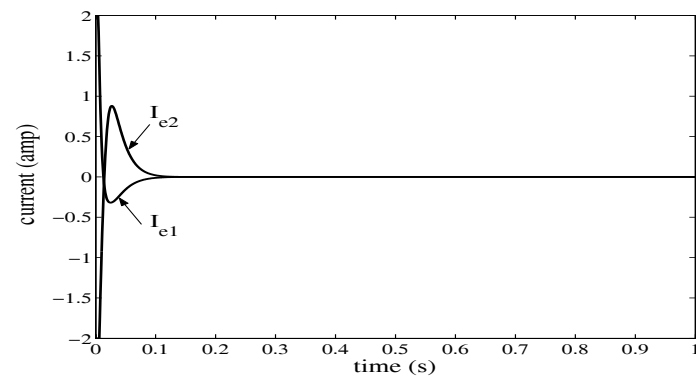

Fig. 8. Stabilization currents 1 and 2 without load disturbances

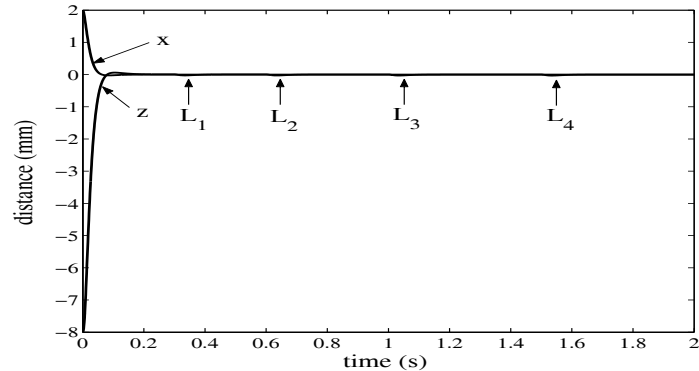

Fig. 9. The carrier motion with load disturbances

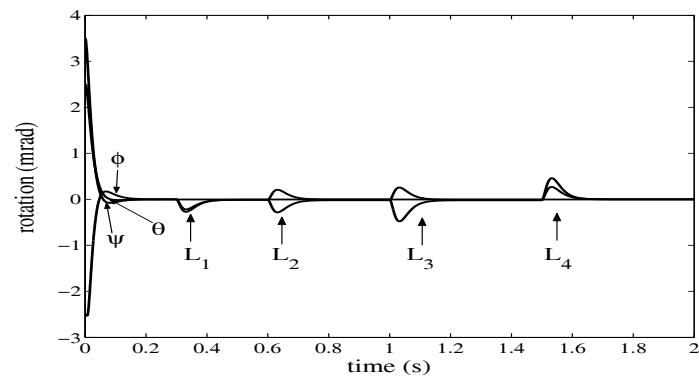

Fig. 10. Carrier rotations with load disturbances

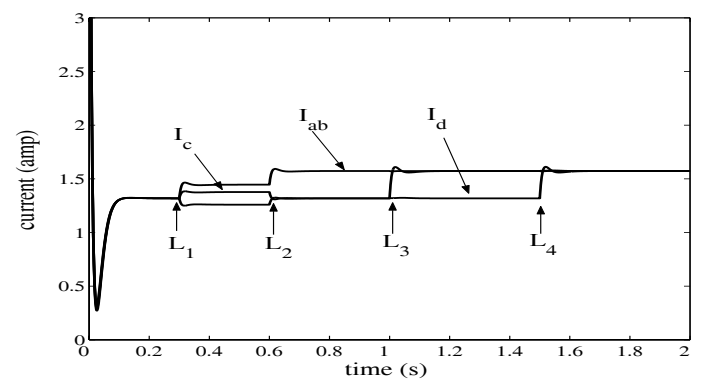

Fig. 11. Behavior of the levitation A, C and D currents

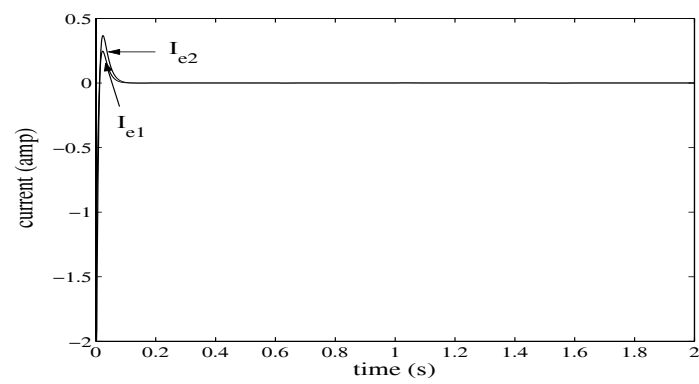

Fig. 12. Behavior of the stabilization 1 and 2 currents 